\title{
LOS PROGRAMAS EDUCATIVOS Y SUS LOGROS
}

\author{
Educational programs and their achievements
}

\author{
Alderete Callupe, Liz $^{1}$ \\ Universidad Nacional del Centro del Perú
}

\begin{abstract}
Resumen
La presente es una investigación documental y sistemática de artículos científicos e investigaciones de bases de datos representativas como Dialnet, Scielo y Scopus para hacer una descripción del estado del Arte de los programas educativos en relación a sus logros. Utilizó como instrumento de recolección de información una matriz de selección de investigaciones y una ficha analítica de investigación, teniendo en cuenta las categorías propuestas y logros. La información fue sistematizada por la hermenéutica como elemento de estructuración fundamentado en referencias conceptuales. Se aprecian investigaciones en procesos educativos y áreas temáticas. Los programas educativos tienden a ocuparse de la Educación, manejo y cuidado, Salud y desempeño empresarial y se dirigen a los niveles Primaria, Secundario y Universitario aplicando metodologías y filosofías, se dirigen también a los campos administrativos, gestión humana e institucional aportando con la aplicación de Estrategias de Motivación y Desempeño profesional. Los logros de los programas educativos son la influencia de la emoción del individuo con sus efectos de regulación socioemocional, mejora de conocimientos en áreas de conducta, determinar y combatir factores negativos y potenciar factores positivos, la mejora académica.

Palabras claves: Ayuda educativa, Proyecto de educación, Programas de educación, Programas educativos de adultos, Ventaja comparativa.
\end{abstract}

\begin{abstract}
This is a documentary and systematic research of scientific articles and research from representative databases such as Dialnet, Scielo and Scopus to make a description of the state of the art of educational programs in relation to their achievements. A research selection matrix and a research analytical sheet were used as an instrument for collecting information, taking into account the proposed categories and achievements. The information was systematized by hermeneutics as a structuring element based on conceptual references. Research in educational processes and thematic areas is appreciated. The educational programs tend to deal with Education, management and care, Health and business performance and are directed to Primary, Secondary and University levels applying methodologies and philosophies, they are also directed to administrative fields, human and institutional management contributing with the application of Motivation and Professional Performance Strategies. The achievements of the educational programs are the influence of the individual's emotion with its effects of socioemotional regulation, improvement of knowledge in behavioral areas, determining and combating negative factors and enhancing positive factors, academic improvement.
\end{abstract}

Keywords: Educational assistance, Educational project, Educational programs, Educational programs for adults, Comparative advantage.

\section{Introducción}

El presente describe el estado del arte de los programas educativos en relación a sus logros para

\footnotetext{
${ }^{1}$ Correspondencia: Liz Alderete Callupe, liz.alderete.c@gmail.com
} 
establecer las bases necesarias en el desarrollo de investigaciones educativas que proponen la implementación, mejora y evaluación de los programas educativos.

El objeto de estudio es la revisión sistemática de artículos científicos e investigaciones en la base de datos Dialnet, Scielo y Scopus, además de repositorios de universidades. El estudio se justifica por la aplicación de las diferentes herramientas teóricas, haciendo comparación importante para la ciencia y el conocimiento científico.

Para confrontar al problema de conocimiento del estado actual de las investigaciones en este campo tan importantes, el logro de aprendizajes es el centro de la educación actual, pues evidencia resultados del proceso enseñanza aprendizaje, de esta forma el camino de la mejora continúa, urge las propuestas creativas de potenciación de habilidades.

Las naciones enfrentan este problema de forma diferente, así las escuelas Norte americanas y las de Europa dictan las clases de acuerdo la propuesta de David Goleman sobre sus habilidades sociales y control de emociones, potenciando el desarrollo de la empatía, autocontrol, autoestima y cooperación, reflejando en los estudiantes menos agresividad y control propio (Bocanegra Malaga, 2019). En el Perú la organización y la planificación de la educación se realizan por medio de los currículos (Ministerio de Educación, 2016; Universidad Nacional del Centro del Perú, 2019).

Cada Estado y organización dentro de su país enfrenta diferentes factores que impiden el conocimiento van desde el manejo deficiente de los alimentos y cuidado ambiental, (Catacora Flores, 2018),y la comorbilidad estudiantil (Orrego Gonzáles, 2016), motivación (Solano Luengo, 2015) hasta la pobre oferta de programas educativos de calidad (Ramos Morales, 2017) es así que se encuentran programas educativos con poca imagen coherente y consistente entre su creación, ejecución y evaluación (Diaz Placencia \& Gamarra Sanchez, 2016).

Un programa educativo es un conjunto de actividades a realizar en lugares y tiempos y recursos determinados previamente para el logro de objetivos y metas, el programa seleccionado debe ser la mejor alternativa de solución previo estudio y diagnóstico de la situación problemática (Núñez Arteaga, 2008).

Los programas educativos son propuestas económicas a los problemas actuales de inserción laboral de los estudiantes sin dejar de lado la mejora de capacidades en competencias de emprendimiento (Palomares-Montero et al., 2019). Cada estudiante que logra suficiencia es un referente para la sociedad (Alva, 2017).

Las actividades que se desarrollan en los talleres permiten adquirir los insumos y su aplicación en el aula, responden a las necesidades que los docentes perciben en aula (Palomares-Montero et al., 2019). Pero los programas educativos abarcan zonas como la salud (Ramos Morales, 2017) en prevención y tratamiento (Catacora Flores, 2018; Ramos García, 2019), campos de emprendimiento protagónico (Martínez García et al., 2018) e inevitablemente al rendimiento académico como indicadores del rendimiento (Gimeno Sacristan, 1976) y variabilidad de este (Alvaro, 1990; Brengelmann, 1975; Edel Navarro, 2003; Martinez Igreda \& Tullume Casas, 2017).

Los programas educativos permiten optimizar los factores que intervienen en el éxito de los emprendimientos, así como la calidad de la idea y del emprendedor, el conocimiento del negocio que se va a emprender y la formación para sostener aquella idea, así como las características personales la honestidad, reputación, carisma y amabilidad. Y las actitudes a desarrollar (Martínez García et al., 2018).

Vallaeys plantea la sostenibilidad del emprendimiento en autoempleo y empleo de otras personas, en este enfoque el individuo es un valor agregado por su competencia, este desarrolla su entorno económico de manera integral, las instituciones educativas participan activamente en esta formación 
de capacidades, pudiendo implementarse innovaciones como la preincubación, incubación, financiamiento y consolidación de empresas, cada fase orientada a la formalización y valor agregado de competencias (Valle \& Pérez, 2016).

Los programas educativos debelan y contrarrestan los factores de carácter psicológico que influencian en el rendimiento del estudiante escolar, entre ellas la inteligencia, las dimensiones dinámicas de la personalidad y los aspectos motivacionales con el enfoque de la relación con la personalidad y motivación descartando las lesiones cerebrales como factores influyentes del rendimiento o éxito, con la posibilidad de modificar la conducta y el rendimiento académico (Mathieux \& antonio Mendoza-Parra, 2016; Suarez Riberiro \& Fernandez, 2019).

Cada familia tiene un nivel socioeducativo, los controles sobre hábitos de estudio y la implicación de la esta misma en actividades escolares (Gaxiola \& Armenta, 2016).

Los programas incluyen también a los padres en actividades extraescolares, ya que su participación potencia la dupla docente - familia (Vilcas, 2017), considera al individuo como catalizador de las experiencias interpersonales, medio ambiente y estudiante en sus entornos familiar, educativo y social.

El ambiente escolar muestra aspectos significativos como la conducta del docente, la condición del estudiante, expectativas de aceptación o rechazo y la notoriedad en el grupo social, metodologías educativas, sistemas de evaluación (De la Fuente Arias et al., 2017). El entorno social incluye a los amigos y sus interrelaciones sociales, las redes sociales, el entorno tanto físico como sociológico. La aceptación frente a sus compañeros de clase es uno de los factores que más influencia (Odiaga Ríos, 2019; Solorzano Torres et al., 2016), ámbitos de intervención educativa programática.

\section{Métodos}

El presente estudio es una investigación documental sistemática de artículos científicos e investigaciones en la base de datos Dialnet, Scielo y Scopus siendo la unidad de análisis cada investigación relevante.

Utilizó como instrumento de recolección de información una matriz de selección de investigaciones y una ficha analítica de investigación, teniendo en cuenta las categorías de: propuestas y logros, la búsqueda se realizó con los booleanos or y and desde las páginas de búsqueda Dialnet, Scielo y Scopus hasta la fecha de 30/05/2021 con las palabras clave: programa + "or" / "and"+ educativo. Para tener una búsqueda eficiente de documentos se inhabilitaron los cortafuegos y antivirus de protección en tiempo real.

Los datos se sistematizaron mediante la hermenéutica como elemento de estructuración. El análisis e interpretación se fundamenta en referencias conceptuales para reconstruir la información y crear propuestas de transformación socio educativo.

Para disgregar los trabajos sin relación al tema se aplicó la matriz de selección de investigaciones, haciendo de esta forma la calificación respecto al área temática.

Luego se hizo el análisis teniendo en cuenta las categorías de propuestas y logros.

La recolección de datos se desarrolla mediante la elaboración de la ficha de recolección de investigaciones en programas educativos. Analizados para definir su consistencia interna basada en la hermenéutica mediante la matriz de análisis de investigaciones educativas.

\section{Resultados}

A través de Dialnet se tuvo acceso a 17946 documentos, 11492 artículos de revista, 3371 tesis, 2714 artículos de libro y 369 libros. En la base de datos Scielo se tienen 934 documentos, de entre ellos 885 artículos originales y 14 de revisión, 480 desde el año 2015, habiéndose identificado de Perú solo 21. En la base de datos Scopus se obtuvo 172 documentos, desde el 2015 solo 90, esta base de datos hace 
conteo por documentos Open Access (71), Gold (62), Hybrid Gold (5), Bronze (3) y Green (40), de estos en total son 160 artículos.

Haciendo la revisión y clasificación se filtraron 11 documentos bajo el criterio de la Ficha de recolección de datos de investigaciones en programas educativos donde se puede apreciar las investigaciones en procesos educativos y las áreas temáticas.

Respecto a esta clasificación se pudo notar investigaciones en programas educativos dirigidos a la Educación primaria, Nivel secundario, Nivel universitario, Aplicación de metodología, Cuidado escolar, Manejo alimenticio, Salud bucal, Estrategias de Motivación y Desempeño profesional. Dejando en claro que los programas educativos tienen aplicaciones diversas. Así se llega a las categorías de Educación, manejo y cuidado, Salud y desempeño.

Las áreas temáticas a la que se aplican los programas educativos es diversa, se tiene de esta forma la aplicación de la Teoría de Daniel Goleman, de igual manera al tema de conocimientos y conductas, la evaluación basada en el desempeño, acompañamiento escolar y a la salud como el mejoramiento de la salud bucal, análisis de actos humanos, manejo alimenticio y el dolor, en la prevención de cáncer de cuello uterino y de mamas, así como en áreas administrativas y sociales como las estrategias motivacionales. Así las categorías se dividen en niveles de educación como de salud o empresarial.

\section{Discusión}

De los documentos filtrados desde la base de datos Dialnet, Scielo y Scopus, bajo el criterio de la Ficha de recolección de datos de investigaciones en programas educativos se aprecian las investigaciones en procesos educativos y las áreas temáticas.

Respecto a esta clasificación se pudo notar que las investigaciones que cumplen con los criterios respecto a programas educativos se dirigen a la enseñanza de la Educación Primaria, Nivel Secundario y Nivel Universitario, niveles donde se pretende ofrecer currículos exigentes con conocimientos científicos y tratando de aportar en el nivel superior con esta de forma activa (Minedu, 2014; Ministerio de Educación, 2016; Unesco-lesalc, 2008)

Los programas educativos tienen lugar en la aplicación de metodologías como en Estados Unidos (Bocanegra Malaga, 2019), existen otros programas educativos con aplicaciones como la de (Nebrija, 2016) que cuenta con su propia filosofía académica y otras como la de (Castaño Madroñal \& Florido del Corral, n.d.; Ramos, 2013) que describen y aplican las metodologías activas de tendencia actual y muy preponderantes para el alcance máximo del proceso enseñanza aprendizaje (UNESCO, 2007; Villa Sánchez, 2018).

A nivel sanitario los programas educativos tomaron un lugar importante para la prevención y tratamiento de enfermedades patológicas y mórbidas así se exalta la aplicación en el cuidado escolar, Manejo alimenticio y Salud bucal entre otros (Gómez del Castillo \& Álvarez, 2002; Organización Panamericana de la Salud, 2014) educación integral (GONZÁLEZ et al., 1993)

A nivel de administración y gestión humana e institucional se aplican los programas educativos también junto a Estrategias de Motivación y Desempeño profesional (Martínez García et al., 2018; Tolentino Sifuentes, 2014) trascendiendo su importancia a los campos institucionales (Orozco Cruz et al., 2009; Palomares-Montero et al., 2019) para tener profesionales y emprendedores de calidad (Camisón, C., Cruz, Gonzáles, 2006; Saquicuya et al., 2019).

A nivel escolar y de formación profesional se aplican los programas especialmente en el factor rendimiento académico pues es el factor psicopedagógico más relevante en estudiantes y docentes (Inuma Macayo \& Seclen Medina, 2019).

Dejando en claro que los programas educativos tienen aplicaciones diversas. 
Así se llega a las categorías de Educación, manejo y cuidado, Salud y desempeño.

Las áreas temáticas a la que se aplican los programas educativos es diversa, se tiene de esta forma la aplicación de la Teoría de Daniel Goleman, de igual manera al tema de conocimientos y conductas, la evaluación basada en el desempeño, acompañamiento escolar y a la salud como el mejoramiento de la salud bucal, análisis de actos humanos, manejo alimenticio y el dolor, en la prevención de cáncer de cuello uterino y de mamas, así como en áreas administrativas y sociales como las estrategias motivacionales. Así las categorías se dividen en niveles de educación como de salud o empresarial.

Los programas educativos no deben enmarcarse solamente al proceso educativo si no ofrecer propuestas económicas y políticas a los problemas actuales (Alva, 2017; Giroux, 2013).

\section{Logros de los programas educativos}

Los programas educativos lograron influencia predominante de la emoción o inteligencia emocional que impulsa la dimensión de autoconocimiento, autorregulación, automotivación, empatía y habilidades sociales (Catacora Flores, 2018). Y relaciones de autorregulación metacognitiva por refuerzo con estrategias motivacionales (Suarez Riberiro \& Fernandez, 2019).

Los programas educativos lograron eficacia en la mejora de los conocimientos y conductas sanitarias (Diaz Placencia \& Gamarra Sanchez, 2016) además de la salud bucal (Martinez Igreda \& Tullume Casas, 2017). Cuidados de manejo alimenticio y manejo de dolor en pacientes de patologías extremas como el cáncer (Orrego Gonzáles, 2016; Ramos García, 2019).

Influencia en el incremento de conocimientos en los temas de primeros auxilios, prevención de accidentes, alimentación, salud y cuidados generales de menores (Ramos Morales, 2017).

Los programas educativos logran determinar los factores relacionados con las características y rasgos personales que se vinculan al éxito de los proyectos, entre ellos la motivación, dedicación, ilusión, tiempo y esfuerzo (Martínez García et al., 2018), además de mejorar el involucramiento en la formación de hábitos de estudio y participación en actividades, deportivas, culturales y sociales (Vilcas, 2017) Estas ofrecen ventajas competitiva ante las actuales tendencias del futuro profesional (Elizabeth AldanaRivera et al., 2019) y dan herramientas necesarias para combatir la falta de recursos económicos, el miedo al fracaso, la inexperiencia y la falta de motivación y evitar el fracaso de emprendimiento (Bombón Remache \& Peñafiel Ramos, 2020).

Los programas educativos logran mejora académica de modos flexibles, dinámicos y entretenidos virtual (Odiaga Ríos, 2019) y presencialmente. Aunque se debe reflexionar en los cambios profundos de los programas académicos donde el docente debe acercarse más al contexto social del estudiante y su sociedad además de repensar en un entorno educativo que integre al estudiante y no lo convierta solo en un repetidor de saberes (Mendoza Castillo, 2020).

Es necesario precisar que los programas educativos deben tener relación con las políticas e identificación de escenarios que permitan el desarrollo de acciones de protección ciudadana en cada aspecto social (Arredondo et al., 2014) desde el financiamiento ya que debemos de superar el $2 \%$ del PBI destinado a salud a nivel nacional para llegar a por lo menos el nivel de Argentina, Colombia y hasta el mismo Cuba donde se invierte mínimamente el $4 \%$ del PBI (Arriagada et al., 2005), aunque es claro que los programas deberían de priorizar la identificación de necesidades de capacitación, elaboración del Diseño Curricular, ejecución de la Educación Sanitaria y la evaluación del Proceso Educativo para garantizar su efectividad (APRISABAC, 1997).

\section{Conclusiones}


Los programas educativos son empleados en múltiples funciones y áreas temáticas, sin embargo, la tendencia de los programas educativos de acuerdo a la ficha de recolección de investigaciones en programas educativos obedecen a las categorizaciones de Educación, manejo y cuidado, Salud y desempeño empresarial.

Respecto a la dirección de los programas educativos se resalta la aplicación escolar a los niveles Primaria, Secundario y Universitario. Se utilizan eficientemente en la aplicación de metodologías y filosofías además en los campos administrativos, gestión humana e institucional los programas aportan con la aplicación de Estrategias de Motivación y Desempeño profesional.

Los logros de los programas educativos son principalmente la influencia de la emoción del individuo con sus efectos de regulación socioemocional, además de la mejora de conocimientos en las áreas de conducta.

Logra también determinar y combatir factores negativos y potenciar factores positivos relacionados con los rasgos personales para tener éxito en proyectos y estilos de vida personal y profesional.

Los programas logran de forma eficiente y muy extendida la mejora académica, aunque para su mejor extensión y aplicación deberán de tener relación con las políticas de turno.

\section{Referencias}

Alva, R. C. (2017). Relación del clima organizacional con el desempeño de los comisarios, comisarías tipo "A" de la VII DIRTEPOL - año 2012. Universidad Nacional Mayor de San Marcos.

Alvaro, M. (1990). Hacia un modelo causal del rendimiento académico (Issue January 2017).

APRISABAC. (1997). Manual de Educación Sanitaria. In Manual de Educación Sanitaria.

Arredondo, A., Reyes, G., \& Moyao, R. (2014). Ventajas y desventajas de los servicios de salud bajo la perspectiva del tratado de libre comercio entre México y Estados Unidos de América. Horizonte Sanitario, 12(3), 84. https://doi.org/10.19136/hs.v12i3.72

Arriagada, I., Aranda, F., \& Divisi, M. (2005). Políticas y programas de salud en América Latina. Problemas y propuestas. In CEPAL - SERIE Políticas sociales (Vol. 114).

http://www.eclac.org/publicaciones/xml/7/23777/sps114_Icl2450.pdf

Bocanegra Malaga, R. J. (2019). Programa educativo basado en la teoría de Daniel Goleman para mejorar la inteligencia emocional de los niños de 5 años de la Institución Educativa Privada Jean Piaget de Nuevo Chimbote 2015 (Vol. 1). Universidad Nacional del Santa.

Bombón Remache, D. E., \& Peñafiel Ramos, V. L. (2020). Los emprendimientos como modalidad de titulación: un diagnóstico de las universidades de la zona 3 del Ecuador. 41(28), 1-12. https://repositorio.uta.edu.ec:8443/jspui/handle/123456789/31813

Brengelmann, J. (1975). Determinantes personales del rendimiento escolar. symposium sobre apredizaje y modificación de conducta en ambientes educativos.

Camisón, C., Cruz, Gonzáles, T. (2006). Gestión de la calidad: Conceptos, enfoques, modelos y sistemas. In Hall, PEARSON Prentice.

Castaño Madroñal, Á., \& Florido del Corral, D. (n.d.). Aplicación de metodologías de enseñanza-aprendizaje basadas en proyectos en antropología social.

Catacora Flores, N. L. (2018). Eficacia de un aprograka acerca de fasciolasis humana, sobre los niveles de conocimientos y conductas en escolares del nivel secundario en el distrito de Asillo, 2017 [Universidad 
San Agustin de Arequipa]. In Universidad San Agustin de Arequipa.

http://repositorio.unsa.edu.pe/bitstream/handle/UNSA/6925/EDMcccacm.pdf?sequence=3\&isAllowed= y

De la Fuente Arias, J., Peralta, F. J., \& Sánchez Roda, M. D. (2017). Valores sociopersonales y problemas de convivencia en la Educación Secundaria. Electronic Journal of Research in Education Psychology, 3(7). https://doi.org/10.25115/ejrep.v4i9.1191

Diaz Placencia, J. A., \& Gamarra Sanchez, J. E. (2016). Impacto educativo de un programa de evaluación basada en el desempeño en los residentes de medicina del programade segunda especialización de la Universidad Privada Antenor Orrego, años 2012-2014 [Universidad Nacional del Trujillo]. http://dspace.unitru.edu.pe/bitstream/handle/UNITRU/3801/Tesis

Edel Navarro, R. (2003). El rendimiento académico: concepto, investigación y desarrollo. REICE. Revista Iberoamericana Sobre Calidad, Eficacia y Cambio En Educación, 1, 2,3-4.

http://www.ice.deusto.es/rinace/reice/vol1n2/Edel.pdf

Elizabeth Aldana-Rivera, E., Tafur-Castillo, J., Gil, I., \& Mejía, C. (2019). Práctica pedagógica de emprendimiento en docentes de educación superior en Institución Educativa Universitaria de Barranquia. Pedagogical Practice of Entrepreneurship in Higher Education Teachers in University Educational Institution of Barranquilla., 38(2), 9-18.

http://search.ebscohost.com/login.aspx?direct=true\&db=a9h\&AN=137697623\&lang=es\&site=ehost-live

Gaxiola, M. I. B., \& Armenta, M. F. (2016). Factores que influyen en el desarrollo y rendimiento escolar de los jóvenes de bachillerato. Revista Colombiana de Psicologia, 25(1), 63-82.

https://doi.org/10.15446/rcp.v25n1.46921

Gimeno Sacristan, J. (1976). Autoconceptor sociabilidad rendimiento escolar.

Giroux, H. (2013). Los profesores como intelectuales. In Journal of Petrology (Vol. 369, Issue 1). https://doi.org/10.1017/CB09781107415324.004

Gómez del Castillo, M., \& Álvarez, M. (2002). Software educativo y educación para la salud. Software Educativo y Educación Para La Salud, 10(19), 179-183. https://doi.org/10.3916/25501

GONZÁLEZ, I. D., Martina HERNÁNDEZ, DÍAZ, L., \& BECERRA, J. (1993). Educación para la salud. Una estrategia integradora. Perfiles Educativos, 62, 42-48.

Inuma Macayo, J. P., \& Seclen Medina, A. (2019). Rendimiento académico en el nivel superior. Universidad Científica del Perú.

Martínez García, I., Padilla Carmona, M. T., \& Suárez Ortega, M. (2018). Aplicación de la metodología Delphi a la identificación de factores de éxito en el emprendimiento. Revista de Investigación Educativa, 37(1), 129-146. https://doi.org/10.6018/rie.37.1.320911

Martinez Igreda, A., \& Tullume Casas, E. (2017). Efectividad del programa educativo Sonrisas Brillantes en el mejoramientode la salud bucal en escolares - Nuevo Chimbote, 2016 [Universidad Nacional del Santa]. http://repositorio.uns.edu.pe/bitstream/handle/UNS/2557/23177.pdf?sequence=1\&isAllowed=y Mathieux, E., \& antonio Mendoza-Parra, M. (2016). We are IntechOpen, the world's leading publisher of Open Access books Built by scientists, for scientists TOP 1 \%. Intech, i(tourism), 13. 
https://doi.org/http://dx.doi.org/10.5772/57353

Mendoza Castillo, L. (2020). Lo que la pandemia nos enseñó sobre la educación a distancia. Revista

Latinoamericana de Estudios Educativos, 50(ESPECIAL), 343-352.

https://doi.org/10.48102/rlee.2020.50.ESPECIAL.119

Minedu. (2014). Marco Curricular Nacional. 1-87.

Ministerio de Educación. (2016). Currículo Nacional de la Educación Básica.

http://www.minedu.gob.pe/curriculo/pdf/curriculo-nacional-de-la-educacion-basica.pdf

Nebrija, G. C. (2016). Metodología de enseñanza y para el aprendizaje. Global Campus Nebrija, 134.

https://www.nebrija.com/nebrija-global-campus/pdf/metodologia-ensenanza-aprendizaje.pdf

Núñez Arteaga, Á. L. (2008). Métodos activos y PNL aplicados en la enseñanza formal. Revista Iberoamericana, $2,1-32$.

Odiaga Ríos, L. M. (2019). Programa Educativo MORALTIC para análisis de actos humanos en universitarios.

EDUCARE ET COMUNICARE: Revista de Investigación de La Facultad de Humanidades, 2(11), 104-118. https://doi.org/10.35383/educare.v2i11.202

Organización Panamericana de la Salud. (2014). Educación para la salud con enfoque integral. Concurso de Experiencias Significativas de Promoción de La Salud En La Región de Las Américas, 3.

http://revistadecomunicacionysalud.org/index.php/rcys/article/view/35/42

Orozco Cruz, J. C., Olaya Toro, A., \& Villate Duarte, V. (2009). ¿Calidad de la educación o educación de calidad? Una preocupación más allá del mercado. Revista Iberoamericana de Educación, 51, 161-181.

https://doi.org/10.35362/rie510637

Orrego Gonzáles, M. E. (2016). Efectividad del programa caminando con el cáncer en el nivel de conocimientos sobre el manejo alimenticio y el dolor, por cuidadores de pacientes de la unidad oncológica del Hospital Regional Docente las Mercedes, 2016. Universidad Señor de Sipan.

Palomares-Montero, D., Chisvert-Tarazona, M. J., \& Suárez-Ortega, M. (2019). Formación y orientación para el emprendimiento. lo que dicen la bibliometría y los emprendedores noveles. REOP - Revista Española de Orientación y Psicopedagogía, 30(1), 131. https://doi.org/10.5944/reop.vol.30.num.1.2019.25198

Ramos, G. A. de L. (2013). La metodología activa en el proceso de enseñanza-aprendizaje y la fundamentación de los estilos de aprendizaje en las alumnas de magisterio de educación infantil. Universidad de San Carlos de Guatemala Escuela.

Ramos García, J. M. (2019). Efectividad de un programa educativo de prevención de cancer de cuello uterino y de mamas en el nivel de conocimiento de estudiantes de la facultad de obstetricia, Unheval, huancuco2018. Universidad Nacional Hermilio Valdizan.

Ramos Morales, B. A. (2017). Influencia del programa educativo "creciendo saludable" en el nivel de conocimiento de la madre sobre el cuidado del niño menor de tres años. Programa Cunamas - Oyotun 2016. Instacron:USS.

Saquicuya, J., Urgilez, C., \& Lopez, M. (2019). Perspectiva de los docentes sobre la escuela de calidad. Espacios, 40(39), 7. https://www.revistaespacios.com/a19v40n39/a19v40n39p07.pdf

Solano Luengo, L. O. (2015). Rendimiento Académico De Los Estudiantes Con Las Aptitudes Mentales y Las 
actitudes ante el estudio [UNED]. In UNED. https://dialnet.unirioja.es/servlet/tesis?codigo=47205

Solorzano Torres, F., Narvaez, A., Alvarado Villa, G., Silva Calle, X., Guerrero Solorzano, J., Poveda Icaza, X., Cabrera Donoso, D., Donoso, D., Perafan Javela, K., Santana Torres, J., Ledesma, P., Torres, S., Dib Fayad, S., Borbor, M., Romero, E., \& Proaño, C. (2016). La familia y los amigos como riesgo de consumo de alcohol en adolescentes / Family and friends as risk for alcohol consumption in adolescents. Ciencia Unemi, 9(17), 85. https://doi.org/10.29076/issn.2528-7737vol9iss17.2016pp85-91p

Suarez Riberiro, J., \& Fernandez, A. (2019). Un modelo sobre cómo las estrategias motivacionales relacionadas con el componente de afectividad inciden sobre las estrategias cognitivas y metacognitivas. Educación XXI, 231-246. https://doi.org/10.5944/educxx1

Tolentino Sifuentes, L. A. (2014). Desempeño didáctico y académico del docente relacionado a la satisfacción de los estudiantes del programa de complementación pedagógica de la Universidad Nacional Mayor de San Marcos, 2013 - II [Universidad Nacional Mayor de San Marcos]. In Universidad Nacional Mayor de San Marcos.

https://doi.org/http://cybertesis.unmsm.edu.pe/bitstream/handle/cybertesis/3981/Tolentino_sl.pdf?se quence $=1$

Unesco-lesalc. (2008). Educación Superior y Sociedad. Educación Superior y Sociedad, 13(2), 206.

UNESCO. (2007). Informe sobre tendencias sociales y educativas en América Latina 2007. In 運輸と経済(Vol. 67, Issue 6).

Universidad Nacional del Centro del Perú. (2019). Prospecto de maestrías y doctorados 2019-II (pp. 1-74). Valle, Y., \& Pérez, C. M. (2016). La Responsabilidad Social Universitaria: emprendimiento sostenible como impacto de intervención en comunidades vulnerables / Educational leadership in the 21st century from the perspective of sustainable entrepreneurship / A Responsabilidade Social Univer. Revista EAN, O(81), 91-110. https://doi.org/10.21158/01208160.n81.2016.1560

Vilcas, L. M. (2017). Acompañamiento escolar de hijos por sus madres que trabajan en el Ministerio de Salud del Distrito de Huancayo [Universidad Nacional del Centro del Perú]. In Horizonte de la Ciencia (Vol. 7, Issue 13). https://doi.org/10.26490/uncp.horizonteciencia.2017.13.362

Villa Sánchez, A. (2018). Tendencias actuales de las transformaciones de las universidades en una nueva sociedad digital. http://www.foroinnovacionuniversitaria.net/tendencias-actuales/ 